\title{
The covariance structure of multifractional Brownian motion, with application to long range dependence
}

\author{
A. Ayache, S. Cohen \\ UFR MIG, Laboratoire de Statistiques et de Probabilités \\ 118, Route de Narbonne, 31062 Toulouse - France \\ and \\ J. Lévy Véhel \\ Projet Fractales, INRIA Rocquencourt, 78153 Le Chesnay Cedex, France
}

\begin{abstract}
Multifractional Brownian motion $(\mathrm{mBm})$ was introduced to overcome certain limitations of the classical fractional Brownian motion ( $\mathrm{fBm})$. The major difference between the two processes is that, contrarily to $\mathrm{fBm}$, the almost sure Hölder exponent of $\mathrm{mBm}$ is allowed to vary along the trajectory, a useful feature when one needs to model processes whose regularity evolves in time, such as Internet traffic or images. Various properties of $\mathrm{mBm}$ have already been investigated in the literature, related for instance to its dimensions or the statistical estimation of its pointwise Hölder regularity. However, the covariance structure of $\mathrm{mBm}$ has not been investigated so far. We present in this work an explicit formula for this covariance. Since $\mathrm{mBm}$ is a zero mean Gaussian process, such a formula provides a full characterization of its stochastic properties. We briefly report on some applications, including the synthesis problem and the long term structure : in particular, we show that the increments of $\mathrm{mBm}$ exhibit long range dependence under general conditions.
\end{abstract}

\section{Introduction and background}

$\mathrm{mBm}$ was introduced in [4] and [11] as the following generalization of $\mathrm{fBm}$ :

\section{Definition 1}

(Multifractional Brownian Motion, Moving Average Definition)

Let $H:[0, \infty) \rightarrow[a, b] \subset(0,1)$ be a Hölder function of exponent $\beta>0$. For $t \geq 0$, the following random function is called multifractional Brownian motion with functional parameter $H$ :

$W_{H(t)}(t)=\int_{-\infty}^{0}\left[(t-s)^{H(t)-1 / 2}-(-s)^{H(t)-1 / 2}\right] d W(s)+\int_{0}^{t}(t-s)^{H(t)-1 / 2} d W(s)$, 
where $W$ denotes ordinary Brownian motion.

\section{Definition 2}

(Multifractional Brownian Motion, Harmonizable Representation)

Let $H:[0, \infty) \rightarrow[a, b] \subset(0,1)$ be a Hölder function of exponent $\beta>0$. For $t \geq 0$ the following function is called multifractional Brownian motion:

$$
W_{H(t)}(t)=\int_{\mathbf{R}} \frac{e^{i t \xi-1}}{|\xi|^{H(t)+\frac{1}{2}}} d W(\xi)
$$

The equivalence, up to a multiplicative deterministic function, between these two formulations has been proved in [6]. From these definitions, it is easy to see that $\mathrm{mBm}$ is a zero mean Gaussian process whose increments are in general neither independent nor stationary (recall that $\mathrm{fBm}$ has stationary correlated increments for $H \neq 1 / 2)$. When $H(t)=H$ for all $t, \mathrm{mBm}$ is of course just $\mathrm{fBm}$ of exponent $H$.

The main feature of this process is that its Hölder regularity varies in time and is equal to $H(t)$. This is in sharp contrast with $\mathrm{fBm}$, where the almost sure Hölder exponent is constant and equal to $H$. More precisely, the following properties of $\mathrm{mBm}$ are known:

Proposition 1 Assume $\beta>\sup _{t>0} H(t)$. With probability one, for each $t_{0}$, the Hölder exponent at point $t_{0} \geq 0$ of multifractional Brownian motion is $H\left(t_{0}\right)$.

(Recall that the Hölder exponent of a process $X(t)$ at point $s$ is defined as: $\alpha_{X}(s, w)=\sup \left\{\alpha, \lim _{h \rightarrow 0} \frac{X(s+h, w)-X(s, w)}{|h|^{\alpha}}=0\right\}$. A "large" $\alpha_{X}(s, w)$ means that $X$ is smooth at $s$, while irregular behaviour of $X$ at $s$ translates into $\alpha$ close to 0 .)

Proposition 2 Assume $\beta>\sup _{t>0} H(t)$. With probability one, for each interval $[c, d] \subset \mathbf{R}^{+}$, the graph of the $m B m\left(W_{H(t)}(t)\right)_{t \in[c, d]}$ verifies the following property:

$\operatorname{dim}_{H}\left\{W_{H(t)}(t), t \in[c, d]\right\}=\operatorname{dim}_{B}\left\{W_{H(t)}(t), t \in[c, d]\right\}=2-\min \{H(t), t \in[c, d]\}$

In addition, $\mathrm{mBm}$ is asymptotically locally self-similar, in the following sense :

\section{Proposition 3}

$\lim _{\rho \rightarrow 0^{+}} \operatorname{Law}\left\{\frac{W_{H\left(t_{0}+\rho u\right)}\left(t_{0}+\rho u\right)-W_{H\left(t_{0}\right)}\left(t_{0}\right)}{\rho^{H\left(t_{0}\right)}}\right\}_{u \in \mathbf{R}^{+}}=\operatorname{Law}\left\{C_{t_{0}} B_{H\left(t_{0}\right)}(u)\right\}_{u \in \mathbf{R}^{+}}$

where $B_{H\left(t_{0}\right)}(u)$ is an $f B m$ of exponent $H\left(t_{0}\right)$ and $C_{t_{0}}$ is a deterministic function of $t_{0}$. 
Thus, while all the properties of $\mathrm{fBm}$ are governed by the unique number $H$, a whole function $H(t)$ is available in the case of $\mathrm{mBm}$. This is useful in many situations where one needs a fine modeling of real world signals. Let us give two examples. It is well known that the long term correlations of the increments of fBm decay as $j^{(2 H-2)}$, where $j$ is the time lag, resulting in long range dependence when $H>1 / 2$ and antipersistent behavior when $H<1 / 2$. In this respect, $\mathrm{fBm}$ is "degenerated" in some sense : since $H$ rules both ends of the Fourier spectrum, i.e. the high frequencies related to the Hölder regularity and the low frequencies related to the long term dependence structure, it is not possible to have at the same time e.g. a very irregular local behavior (implying $H$ close to 0 ) and long range memory (implying $H>1 / 2$ ). fBm is thus not adapted to model processes which displays both those features, such as Internet traffic. Another example is in the field of image synthesis: $\mathrm{fBm}$ has frequently been used for generating artificial mountains. Such a modeling assumes that the irregularity of the mountain is everywhere the same. It thus does not allow to take into account erosion or other meteorological phenomena which smooth some parts of the mountains more than others. To model these and other fine features of natural landscapes (such as faults), $\mathrm{mBm}$ is a good candidate.

A crucial question for applications is of course that of the estimation of the functional parameter $H(t)$. Obviously, this is a much harder task that in the case of $\mathrm{fBm}$, and one could fear that the added flexibility gained in the modeling thanks to the use of a varying $H$ might be seriously impaired in practice. Fortunately, robust estimators have been proposed in [3], which allow reasonable accuracy on real signals.

As said above, an attractive property of $\mathrm{fBm}$ which has been invoked in many applications is the long range dependence of its increments when $H>1 / 2$. This property was the main motivation for the use of $\mathrm{fBm}$ for instance in financial engineering, traffic modeling, geophysical and physiological time series analysis ... A natural question is to ask whether mBm may also exhibit this feature, depending on the values of the function $H(t)$. This would allow to fulfill our program above, i.e. have a natural generalization of $\mathrm{fBm}$ that permits to control independently the Hölder regularity and the low frequency part of the Fourier spectrum.

A natural way to check for the possible occurrence of long range dependence in a process is to look at the autocovariance. More generally, $\mathrm{mBm}$ being a Gaussian process, all of its stochastic properties can be obtained from its autocovariance. It is thus important to dispose of an explicit formula for it. Although this may seem surprising, such a formula does not seem to have been derived so far. Indeed, most of the efforts in the study of $\mathrm{mBm}$ have been directed towards the investigation of its local regularity (e.g. which functions $H(t)$ are allowed), the dimensional properties of its graph and the estimation of $H(t)$.

The main motivation of this paper is to provide, in section 2, explicit formulae for the autocovariance and autocorrelation of $\mathrm{mBm}$. Section 3 presents some applications of these formulae, mainly for the synthesis of $\mathrm{mBm}$ and for the study of its long range dependence properties. 
Note finally that $\mathrm{mBm}$ was generalized in [2] to allow for a discontinuous $H(t)$. The study of the autocovariance for this generalized $\mathrm{mBm}$ will be presented elsewhere.

\section{Computation of the covariance of $\mathrm{mBm}$}

Our aim in this section is to obtain explicit expressions for the autocovariance and autocorrelation of $\mathrm{mBm}$.

Proposition 4 Let $X(t)$ be a standard $m B m$ (i.e. such that the variance a time 1 is 1) with functional parameter $H(t)$. Then, E denoting expectation,

$\operatorname{cov}_{X}(t, s)=\mathrm{E}(X(t) X(s))=D(H(t), H(s))\left(t^{H(t)+H(s)}+s^{H(t)+H(s)}-|t-s|^{H(t)+H(s)}\right)$

where

$$
D(x, y)=\frac{\sqrt{\Gamma(2 x+1) \Gamma(2 y+1) \sin (\pi x) \sin (\pi y)}}{2 \Gamma(x+y+1) \sin (\pi(x+y) / 2)}
$$

Proof

By definition,

$$
\begin{aligned}
E(X(t) X(s)) & =E\left(\frac{1}{C(H(t))} \int_{\mathbf{R}} \frac{e^{i \xi t}-1}{|\xi|^{H(t)+\frac{1}{2}}} d W(\xi) \frac{1}{C(H(s))} \int_{\mathbf{R}} \frac{e^{-i \xi s}-1}{|\xi|^{H(s)+\frac{1}{2}}} d W(\xi)\right) \\
& =\frac{1}{C(H(t)) C(H(s))} \int_{\mathbf{R}} \frac{\left(e^{i \xi t}-1\right)\left(e^{-i \xi s}-1\right)}{|\xi|^{H(t)+H(s)+1}} d \xi
\end{aligned}
$$

where the value of $C(H(t))=\left(\frac{\pi}{H(t) \Gamma(2 H(t)) \sin \pi H(t)}\right)^{\frac{1}{2}}$ is deduced from the requirement that $E\left(X^{2}(1)\right)=1$.

Fix $t, s$, and let $B_{H}$ be a standard $\mathrm{fBm}$ with fractional parameter $H=$ $\frac{H(t)+H(s)}{2}$. It is well known that:

$$
\begin{aligned}
E\left(B_{H}(t) B_{H}(s)\right) & =\frac{1}{C^{2}} \int_{\mathbf{R}} \frac{\left(e^{i \xi t}-1\right)\left(e^{-i \xi s}-1\right)}{|\xi|^{2 H+1}} d \xi \\
& =\frac{1}{2}\left(t^{2 H}+s^{2 H}-|t-s|^{2 H}\right)
\end{aligned}
$$

where $C=\left(\frac{\pi}{H \Gamma(2 H) \sin \pi H}\right)^{\frac{1}{2}}$.

Thus:

$$
\begin{aligned}
\int_{\mathbf{R}} \frac{\left(e^{i \xi t}-1\right)\left(e^{-i \xi s}-1\right)}{|\xi|^{H(t)+H(s)+1}} d \xi & =C^{2} E\left(B_{H}(t) B_{H}(s)\right) \\
& =\frac{C^{2}}{2}\left(t^{2 H}+s^{2 H}-|t-s|^{2 H}\right)
\end{aligned}
$$


Substituting in (1):

$E(X(t) X(s))=\frac{C^{2}}{2 C(H(t)) C(H(s))}\left(t^{H(t)+H(s)}+s^{H(t)+H(s)}-|t-s|^{H(t)+H(s)}\right)$

Replacing $H$ by $\frac{H(t)+H(s)}{2}$ in $C$ and using the identity $x \Gamma(x)=\Gamma(x+1)$ yields the announced equality.

Note that because $H$ takes values in $[a, b], D(H(t), H(s))$ is positive and stays bounded away from 0 and $\infty$. $\mathrm{mBm}$ being non stationary, it is often more pertinent to consider the autocorrelation $\operatorname{cor}_{X}(t, s)$ :

Proposition 5 Let $X(t)$ be a standard $m B m$ with functional parameter $H(t)$. Then,

$$
\operatorname{cor}_{X}(t, s)=\frac{D(H(t), H(s))\left(t^{H(t)+H(s)}+s^{H(t)+H(s)}-|t-s|^{H(t)+H(s)}\right)}{t^{H(t)} s^{H(s)}}
$$

\section{Proof}

By definition, $\operatorname{cor}_{X}(t, s)=\frac{\operatorname{cov}_{X}(t, s)}{\sqrt{\mathrm{E}\left(X^{2}(t)\right) \mathrm{E}\left(X^{2}(s)\right)}}$. Since $D(H(t), H(t))=\frac{1}{2}$, we get that $\mathrm{E}\left(X^{2}(t)\right)=t^{2 H(t)}$ and the result follows.

Recall that one of our main motivation is to study, by analogy with what is done for $\mathrm{fBm}$, the long range dependence of the increments of $\mathrm{mBm}$. We will thus be also interested in the autocovariance of the increments of $\mathrm{mBm}$, or, more precisely, their autocorrelation, the increments being also non stationary. While Proposition 4 allows to write explicit forms, these do not give much insight, so we will not give them here.

\section{Applications}

\subsection{Synthesis of $\mathrm{mBm}$}

Because $\mathrm{mBm}$ is non stationary with non stationary increments in general, care must be taken when synthesizing its sample paths. Let us first recall a few facts about the generation of sample paths of $\mathrm{fBm}$. Numerous methods have been proposed in this context. They include Choleski decomposition, midpoint displacement and its various improvements [7], spectral synthesis [10], waveletbased methods [12], and synthesis based on differential models [9]. The reason why so many algorithms exist is that synthesizing an $\mathrm{fBm}$ is by no means an easy process, especially if one needs to build large traces. The problem lies mainly in the non-Markovian nature of $\mathrm{fBm}$, the strong correlations that it displays (for $H<1 / 2$, each increment is negatively correlated with all the others, while for $H>1 / 2$ the spectral density of the increments is singular at the origin). As well, it is a recognized fact that all methods, except the Choleski one, are only 
approximate. It is not clear however why the unique exact synthesis, through Choleski decomposition, is not used systematically. It probably stems from the wrong impression that this method is greatly time and memory consuming. While this is true for a plain implementation of the algorithm, several refinements allow to reduce the time and memory requirements to values comparable to the ones of the "fast" approximate methods. Let us describe in some detail the principal steps involved in the Choleski decomposition method.

Assume we wish to generate samples of an $\mathrm{fBm} X$ with exponent $H$ at $N$ equidistant points of $[0,1]$. Let $D X$ denote the discrete increments of $X$, that is $D X(k / N)=X(k / N)-X((k-1) / N), k=1, \ldots N$. These increments form a discrete stationary Gaussian process with zero mean, and the statistical properties of the vector $D X_{N}=(D X(1 / N), D X(2 / N), \ldots, D X(1))$ are entirely determined by the autocovariance matrix $A_{N}=\mathrm{E}\left(D X_{N}\left(D X_{N}\right)^{T}\right)$, where $U^{T}$ denotes the transpose of $U$. It is well known that $\mathrm{E}(D X(i / N) D X((i+k) / N))=$ $\frac{1}{2 N^{2 H}}\left(|k+1|^{2 H}+|k-1|^{2 H}-2|k|^{2 H}\right)$. Since $A_{N}$ is positive definite, it may be written using its Choleski decomposition as:

$$
A_{N}=L_{N} L_{N}^{T}
$$

where $L_{N}$ is an invertible lower triangular matrix.

Let $D Y_{N}=(D Y(1 / N), D Y(2 / N), \ldots, D Y(1))$ be an $N$-samples realization of a unit variance centered white Gaussian noise. It is easy to see that the autocovariance matrix of the random vector $L_{N} D Y_{N}$ is exactly $A_{N}$. Indeed:

$$
\mathrm{E}\left(L_{N} D Y_{N}\left(L_{N} D Y_{N}\right)^{T}\right)=L_{N} \mathrm{E}\left(D Y_{N}\left(D Y_{N}\right)^{T}\right) L_{N}^{T}=A_{N}
$$

We may thus set $D X_{N}=L_{N} D Y_{N}$, and generate a realization of the $\mathrm{fBm} X$ as $X(k / N)=\sum_{p=1}^{k} D X(p / N)$. Since $A_{N}$ depends only on $N$ and $H$, it is entirely determined once we have fixed the exponent and the number of points we wish to generate. The problem of synthesizing a sample of an $\mathrm{fBm}$ is thus reduced to that of computing $L_{N}$ from $A_{N}$. Note that, so far, we have only used the fact that $A_{N}$ is a valid autocovariance matrix, so that it has a Choleski decomposition. Thus the procedure above may be applied for synthesizing any discrete Gaussian process. A direct method for general Choleski factorization has complexity $O\left(N^{3}\right)$ and requires $O\left(N^{2}\right)$ memory. This precludes the use of this approach for building large traces, and is the reason usually invoked for the need of fast approximate methods. However, when the process is stationary (this is why we work with $D X$ rather than $X$ ) and in the common case where the samples are equi-spaced, the matrix considered is Toeplitz: one can then use fast algorithms, such as the Schur or Levinson algorithms, which have complexity $O\left(N^{2}\right)$ and need $O(N)$ memory. Furthermore, it is possible to do even better if one forces $N$ to be a power of 2 . Such a requirement is common to many methods (e.g. FFT or dyadic wavelet-based), and is not generally considered as a major drawback. In this case the doubling Schur algorithm [1] allows to reduce the complexity to $O\left(N\left(\log _{2}(N)\right)^{2}\right)$. This is very reasonable and permits the exact synthesis of quite large traces (for comparison, spectral methods based on the FFT have complexity $O\left(N\left(\log _{2}(N)\right)\right)$. 
Let us now turn to the synthesis of $\mathrm{mBm}$. The usual technique is based on the following theorem ([11]):

Theorem 1 Let $\left(B_{H}(t)\right)_{t \geq 0}$ be an $f B m$ of index $H$. Then for any interval $[a, b] \subset(0,1)$ and $K>0$ we have almost surely

$$
\begin{aligned}
& \lim _{h \rightarrow 0} \sup _{a \leq H, H^{\prime} \leq b} \sup _{t \in[0, K]}\left|B_{H}(t)-B_{H^{\prime}}(t)\right|=0 . \\
& \left|H^{\prime}-H\right|<h
\end{aligned}
$$

This result allows to generate a sample path of an $\operatorname{mBm} X(t)$ with $t \in\left[t_{0}, t_{1}\right]$ through the following procedure :

- Denote $H_{i}=H\left(t_{i}\right)$ for $t_{i}=t_{0}+\left(t_{1}-t_{0}\right) \frac{i}{N}, i=0 \ldots N$, where $N+1$ is the number of sample points to be generated.

- For a fixed $\omega$, synthesize all fBm-s $B_{H_{i}}$ with parameter $H_{i}$ on $\left[t_{0}, t_{1}\right]$. More precisely, we generate one realization of a white noise $D Y_{N}$ and compute all the matrices $L_{N}\left(H_{i}\right)$ corresponding to the increments of fBm-s with exponent $H_{i}$, as defined in (2). We then set $D X_{N}^{H_{i}}=L_{N}\left(H_{i}\right) D Y_{N}$ and add these increments to obtain $B_{H_{i}}$.

- $X$ is then obtained by setting $X\left(t_{i}\right)=B_{H_{i}}\left(t_{i}\right)$.

This procedure has complexity $N$ times the complexity of generating a single $\mathrm{fBm}$, and needs $N$ times the memory required for the synthesis of an $\mathrm{fBm}$. Thus, if one uses the Schur algorithm, the time complexity is $O\left(N^{3}\right)$ and $O\left(N^{2}\right)$ memory is needed. In the case the doubling Schur algorithm is used, the time complexity falls to $O\left(N^{2}\left(\log _{2}(N)\right)^{2}\right)$.

Now that we dispose of a formula for the autocovariance of $\mathrm{mBm}$, an alternate method may be proposed : one can use the Choleski decomposition to generate directly the samples of an $\mathrm{mBm}$, without synthesizing first all the "tangent" fBm-s. Indeed, as said above, the Choleski method may be applied for building traces of any discrete Gaussian process. The new method thus uses as a starting point the matrix $A_{N}$ deduced from Proposition 4, and computes the corresponding matrix $L_{N}$. Note that, in the case of $\mathrm{mBm}$, there is no point in working with the increments : since they are not stationary, it is not simpler to obtain $L_{N}$ for the increments than for the original process. In turn, this imply that one cannot make use of fast algorithms for the decomposition, so that the time (resp. memory) complexity will be $O\left(N^{3}\right)$ (resp. $O\left(N^{2}\right)$ ). We thus obtain exactly the same values as in the previous synthesis method when the Schur algorithm is used. In particular, the new method is worse than the old one when $N$ may be chosen to be a power of 2 . Note however that it is exact, as the previous one was only approximate.

\subsection{Long range dependence}

Let us first recall what is usually meant by "long range dependence". Let $Y(t)$ be a stationary process with $\mathrm{E}\left(Y^{2}(t)\right)=1$. The correlation function of $Y(t)$, 
denoted $\rho$, depends only on the lag :

$$
\rho(k)=\mathrm{E}(Y(t) Y(t+k))
$$

One possible definition of long range dependence is the following [5] :

\section{Definition 3}

Let $Y(t)$ be a stationary process. $Y(t)$ is said to have long range dependence if there exists a number $\alpha \in(-1,0)$ and a constant $c$ such that :

$$
\lim _{k \rightarrow \infty} \frac{\rho(k)}{c k^{\alpha}}=1
$$

Some authors use a less stringent definition, based on the summability of the correlation function :

\section{Definition 4}

Let $Y(t)$ be a stationary process. $Y(t)$ is said to have long range dependence if $\sum_{0}^{+\infty}|\rho(k)|=+\infty$

The increments of $\mathrm{fBm}$ yield the most well known example of long range dependenceé: it is classical that, for $H \neq \frac{1}{2}, \rho(j)$ is equivalent to $H(2 H-1) j^{2 H-2}$ when $j$ tends to infinity. In addition,

- $0<H<\frac{1}{2}: \sum_{-\infty}^{+\infty} \rho(k)=0$ and $\forall k \neq 0, \rho(k)<0$ (antipersistant behavior)

- $H=\frac{1}{2}: \rho(k)=0, \forall k \neq 0$ (independents increments of Brownian motion).

- $\frac{1}{2}<H<1: \sum_{-\infty}^{+\infty} \rho(k)=+\infty$ (long range dependence)

An alternative definition is based on the behavior of the spectral density at the origin : long range dependence manifests itself through a divergence at low frequencies.

\section{Definition 5}

Let $Y(t)$ be a stationary process. $Y(t)$ is said to have long range dependence if there exists a number $\beta \in(-1,0)$ and a constant $d$ such that :

$$
\lim _{\lambda \rightarrow 0} \frac{f(\lambda)}{d \lambda^{\beta}}=1
$$

where $f$ denotes the spectral density of $Y$. 
Again in the case of the increments of $\mathrm{fBm}$, it is well known that the spectral density reads :

$$
f(\lambda)=2 c(1-\cos \lambda) \sum_{j=-\infty}^{+\infty}(2 \pi j+\lambda)^{-2 H-1}, \lambda \in[-\pi, \pi]
$$

with

$$
c=\frac{\sigma^{2}}{2 \pi} \sin (\pi H) \Gamma(2 H+1)
$$

As a consequence,

$$
f(\lambda)=c|\lambda|^{1-2 H}+O\left(|\lambda|^{\min (3-2 H, 2)}\right) \text { when } \lambda \longrightarrow 0
$$

and :

- $H<\frac{1}{2}: f(\lambda) \lambda \rightarrow 00$.

- $H>\frac{1}{2}: f(\lambda) \lambda \rightrightarrows 0+\infty$.

Of course, these definitions must be adapted in our case, since mBm does not have stationary increments. In particular, it is not straightforward to define a spectral density for the increments : in general, there are several, non equivalent, ways to extend the notion of a Fourier spectrum for non stationary processes. For instance, [8] proposes to compute first the Wigner-Ville transform, which, for a process $X(t)$ yields a function $(t, f) \rightarrow W(t, f)$, where $f$ represents frequency. An average spectrum is then defined by integrating $W(t, f)$ with respect to $t$. This procedure allows, in the case of $\mathrm{fBm}$, to obtain in a satisfactory way the intuitive fact that the spectrum behaves as $1 /|f|^{2 H+1}$. Although it is theoretically possible to follow the same route for $\mathrm{mBm}$, computing the Wigner-Ville spectrum does not seem to be an easy task when $H(t)$ is an arbitrary Hölder function. We will thus rather study the asymptotic behavior of the correlation function of the increments, $\operatorname{cor}_{Y}(t, s)$. This function depends on both time instants, and the best we can do is to fix one "initial" time, say $s$, and see what happens when we let $t$ go to infinity. In doing so, we will obtain an asymptotic behavior conditioned on $s$, reflecting the fact that the long term correlation structure will in general be different for different initial times. In addition, and again because of non stationarity, we cannot hope in general that the ratio $\frac{\operatorname{cor}_{Y}(s, s+h)}{h^{\alpha}}$ will have a non degenerate limit $c$ for a certain well chosen $\alpha$. Rather, we will content ourselves with the fact that $\frac{\operatorname{cor}_{Y}(s, s+h)}{h^{\alpha}}$ stays bounded away from 0 and $\infty$ when $h$ tends to infinity. We thus set the following definitions for long range dependence of non (necessarily) stationary processes :

\section{Definition 6}

Let $Y(t)$ be a second-order process. $Y(t)$ is said to have long range dependence if there exists a function $\alpha(s)$ taking values in $(-1,0)$ such that:

$$
\forall s \geq 0, \operatorname{cor}_{Y}(s, s+h) \approx h^{\alpha(s)}
$$


when $h$ tends to infinity.

$(f(h) \approx g(h)$ when $h$ tends to infinity denotes the property that there exist $0<c<d<\infty$ such that for all sufficiently large $h, c \leq \frac{f(h)}{g(h)} \leq d$.)

By analogy with Definition 4 , we also consider the weaker condition on the summability of the autocorrelation :

\section{Definition 7}

Let $Y(t)$ be a second-order process. $Y(t)$ is said to have long range dependence if

$$
\forall \delta>0, \forall s \geq 0, \sum_{0}^{+\infty}\left|\operatorname{cor}_{Y}(s, s+k \delta)\right|=+\infty
$$

Note finally that since the increments are not stationary, it is important to consider the correlation function rather than the covariance : for stationary processes, these differ by a multiplicative constant, but for $\mathrm{mBm}$ (or its increments), the ratio $\frac{\text { covariance }}{\text { correlation }}$ tends to infinity when $t$ tends to infinity.

From now on, we assume that $H$ is a non constant function.

Proposition 6 (asymptotic behavior of the covariance of $\mathrm{mBm}$ )

Let $X(t)$ be a standard $m B m$ with functional parameter $H(t)$. Then, when $t$ tends to infinity, and for all fixed $s \geq 0$,

$H(t)+H(s)<1 \Rightarrow \operatorname{cov}_{X}(t, s) \approx 1$

$H(t)+H(s)>1 \Rightarrow \operatorname{cov}_{X}(t, s) \approx t^{H(t)+H(s)-1}$

\section{Proof}

Recall that, because $H(u)$ varies in $[a, b]$ with $0<a<b<1$, the renormalising function $D(H(t), H(s))$ takes values in an interval $\left[d_{\min }, d_{\max }\right]$ with $0<d_{\min }<d_{\max }<\infty$. Since we are only interested in the order of magnitude of $\operatorname{cov}_{X}(t, s)$, we may from now on neglect $D(H(t), H(s))$ in our computations. The announced result then follows simply from a Taylor expansion of $\left(t^{H(t)+H(s)}+s^{H(t)+H(s)}-|t-s|^{H(t)+H(s)}\right)$, where the leading term is

$$
D(H(t), H(s)) s^{H(t)+H(s)} \text { if } H(t)+H(s)<1
$$

and

$$
D(H(t), H(s))(H(t)+H(s)) s t^{H(t)+H(s)-1} \text { if } H(t)+H(s)>1 .
$$

(recall that $H(t)+H(s)$ is bounded.)

Proposition 7 (asymptotic behavior of the correlation of $\mathrm{mBm}$ )

Let $X(t)$ be a standard $m B m$ with functional parameter $H(t)$. Then, when $t$ tends to infinity, and for all fixed $s \geq 0$,

$H(t)+H(s)<1 \Rightarrow \operatorname{cor}_{X}(t, s) \approx t^{-\overline{H(}(t)}$

$H(t)+H(s)>1 \Rightarrow \operatorname{cor}_{X}(t, s) \approx t^{H(s)-1}$ 


\section{Proof}

It suffices to recall that the variance at time $t$ of an $\mathrm{mBm}$ is $O\left(t^{2 H(t)}\right)$.

Since both $-H(t)$ and $H(s)-1$ belong to $(-1,0)$ for all $t, s$, we have the following

Corollary 1 For all admissible $H(t), m B m$ has long range dependence in the sense of Definition 7. If, for all $s, H(t)+H(s)>1$ for all sufficiently large $t$, then the $m B m$ associated to $H$ has long range dependence in the sense of Definition 6, with functional long range dependence exponent $\alpha(s)=H(s)-1$.

A simple case where an $\mathrm{mBm}$ does not have long range dependence in the sense of Definition 6 is when there exists a sequence $\left(t_{n}\right)_{n}$ tending to infinity such that $H\left(t_{n}\right)+H(s)<1$ for all $n$ and $H$ does not have a limit. In this case, there does not exist an exponent $\alpha_{s}$ such that $\operatorname{cor}_{Y}(s, s+h)=O\left(h^{\alpha_{s}}\right)$.

Let us now consider the increments of $\mathrm{mBm}$.

Proposition 8 (asymptotic behavior of the covariance of the increments of $m B m$ )

Let $X(t)$ be a standard $m B m$ with functional parameter $H(t)$ and $Y(t)$ be the unit time increments of $X(t)$, i.e. $Y(t)=X(t+1)-X(t)$. Then, when $t$ tends to infinity, and for all fixed $s \geq 0$ such that the four quantities $H(t)+H(s), H(t+$ $1)+H(s), H(t)+H(s+1)$, and $H(t+1)+H(s+1)$ are all different, $\max (H(t)+H(s), H(t+1)+H(s), H(t)+H(s+1), H(t+1)+H(s+1))<1$ $\Rightarrow \operatorname{cov}_{Y}(t, s) \approx 1$

$\max (H(t)+H(s), H(t+1)+H(s), H(t)+H(s+1), H(t+1)+H(s+1))>1$ $\Rightarrow \operatorname{cov}_{Y}(t, s) \approx t^{\max (H(t)+H(s), H(t+1)+H(s), H(t)+H(s+1), H(t+1)+H(s+1))-1}$

\section{Proof}

By definition, $\operatorname{cov}_{Y}(t, s)=\operatorname{cov}_{X}(t+1, s+1)-\operatorname{cov}_{X}(t+1, s)-\operatorname{cov}_{X}(t, s+1)+$ $\operatorname{cov}_{X}(t, s)$. By Proposition 6, if $\max (H(t)+H(s), H(t+1)+H(s), H(t)+H(s+$ 1), $H(t+1)+H(s+1))<1$, all the covariances on the right side above are $O(1)$ and their sum does not cancel if $H(t)+H(s), H(t+1)+H(s), H(t)+H(s+1)$, and $H(t+1)+H(s+1)$ all differ. If at least one of $H(t)+H(s), H(t+1)+$ $H(s), H(t)+H(s+1)$ and $H(t+1)+H(s+1))$ is greater than one, the order of $\operatorname{cov}_{Y}(t, s)$ will be the maximum of these values, since they all differ. More precisely, denoting $(\bar{t}, \bar{s})$ the couple where the maximum of $H(t)+H(s), H(t+$ $1)+H(s), H(t)+H(s+1), H(t+1)+H(s+1)$ is attained, we have that :

$$
\operatorname{cov}_{Y}(t, s)=\bar{s} D(H(\bar{t}), H(\bar{s}))(H(\bar{t})+H(\bar{s})) t^{H(\bar{t})+H(\bar{s})-1}+o\left(t^{H(\bar{t})+H(\bar{s})-1}\right) .
$$

\section{Remark}

Since interesting cases are when $H$ is a non constant function, the condition that $H(t)+H(s), H(t+1)+H(s), H(t)+H(s+1)$, and $H(t+1)+H(s+1)$ are all different is not a strong constraint but rather corresponds to the "generic" case. 
Proposition 9 (asymptotic behavior of the correlation of the increments of $m B m$ )

Let $X(t)$ be a standard $m B m$ with functional parameter $H(t)$ and $Y(t)$ be the unit time increments of $X(t)$. Let $\operatorname{cor}_{Y}(t, s)$ denote the correlation of $Y$. Then, when $t$ tends to infinity, if $H(t)$ and $H(t+1)$ differ, and for all fixed $s \geq 0$ such that the four quantities $H(t)+H(s), H(t+1)+H(s), H(t)+H(s+1)$, and $H(t+1)+H(s+1)$ are all different,

$\max (H(t)+H(s), H(t+1)+H(s), H(t)+H(s+1), H(t+1)+H(s+1))<1$

$\Rightarrow \operatorname{cor}_{Y}(t, s) \approx t^{-\max (H(t), H(t+1))}$

$\max (H(t)+H(s), H(t+1)+H(s), H(t)+H(s+1), H(t+1)+H(s+1))>1$

$\Rightarrow \operatorname{cor}_{Y}(t, s) \approx t^{\max (H(s), H(s+1))-1}$.

\section{Proof}

Again, this is simply obtained using Proposition 8 plus the fact that $\mathrm{E}\left(Y^{2}(t)\right)=$ $O\left(t^{2 \max (H(t), H(t+1))}\right)$ if $H(t)$ and $H(t+1)$ differ (otherwise cancellation occur and the leading term is different). The exponent in the case where $\max (H(t)+$ $H(s), H(t+1)+H(s), H(t)+H(s+1), H(t+1)+H(s+1))>1$ results from the identity :

$\max (H(t)+H(s), H(t+1)+H(s), H(t)+H(s+1), H(t+1)+H(s+1))$

$-\max (H(t), H(t+1))=\max (H(s), H(s+1))$.

We are now in position to answer the question raised in the introduction about the possible long range dependence of the increments of $\mathrm{mBm}$.

Corollary 2 For all admissible $H(t)$, the increments of $m B m$ have long range dependence in the sense of Definition 7. If, for all $s$,

$\max (H(t)+H(s), H(t+1)+H(s), H(t)+H(s+1), H(t+1)+H(s+1))>1$ for all sufficiently large $t$, then the increments of the $m B m$ associated to $H$ have long range dependence in the sense of Definition 6, with functional long range dependence exponent $\alpha(s)=\max (H(s), H(s+1))-1$.

\section{Proof}

Obviously, both $\max (H(s), H(s+1))-1$ and $-\max (H(t), H(t+1))$ belong to $(-1,0)$.

A simple example is described in the following proposition :

Proposition 10 Let $H(t)$ be an increasing Hölder function on $\mathbf{R}^{+}$such that $H([0, \infty))=[a, b)$ with $0<a<b<1$ and $a+b>1$ (e.g. $\left.H(t)=a+(b-a) \frac{t}{1+t}\right)$. Then the increments of the associated $m B m$ displays long range dependence in the sense of Definition 6, i.e.:

$$
\forall s \geq 0, \operatorname{cor}_{Y}(s, s+h) \approx h^{\alpha(s)}
$$

when $h$ tends to infinity, where $\alpha(s)=H(s+1)-1 \in(-1,0)$.

Proof

Since $a+b>1$ and $H$ is increasing, $H(s)+H(t)>1$ for all sufficiently large $t$. 
The conditions set on $H$ allows to apply Proposition 9 to get that : $\operatorname{cor}_{Y}(s, s+h) \approx h^{H(s+1)-1}$

The exponent $\alpha_{s}=H(s+1)-1$ is indeed independent of $h$ and belongs to $(-1,0)$.

Corollary 2 and Proposition 10 show that $\mathrm{mBm}$ is indeed a generalization of $\mathrm{fBm}$ that allows to have at the same time long range dependence in the sense of Definition 7 and arbitrarily low Hölder regularity at all times. In addition, we can choose $H$ so that the associated $\mathrm{mBm}$ has long range dependence in the stronger sense of Definition 6 while having arbitrarily low regularity. More generally, it is worthwhile to note that, as far as long range dependence is concerned, the situation is essentially different from the fBm case : long range dependence is obtained for the full range of admissible values of $H$, and not just for $H \in\left(\frac{1}{2}, 1\right)$.

It is also possible to obtain more "exotic" behaviors : take for instance $H(t)=\frac{1}{3}+\frac{1}{2}\left|\sin \left(\frac{\pi}{2} t\right)\right|$. Then, for some values of the couple (initial time $=$ $s, l a g=k \in \mathbf{Z})$, we shall have that $\operatorname{cor}_{Y}(s, s+2 k)$ satisfies the conditions of Definition 6 , while $\operatorname{cor}_{Y}(s, s+2 k+1)$ does not. Thus, the occurrence of long range dependence will also depend on the time step between consecutive observations, i.e. the unit lag. Using the same kind of ideas, one can design an $H$ such that the associated $\mathrm{mBm}$ has long range dependence on any given measurable subset of $[0, \infty)$, and not on its complementary.

\subsection{Other applications}

There are of course many other ways to exploit Proposition 4. We do no more in this section than sketch some of these possible applications.

Financial modeling :

In recent years, there has been a growing interest in modeling financial time series with $\mathrm{fBm}$ rather than Brownian motion. Such a refinement allows to take into account the strong correlations observed in real traces. Long memory in this framework is crucial in particular as far as efficiency is concerned, because it means that prices will fall back to their fundamental value much more slowly than if the market was without memory. In consequence, it is possible to make profits using this durable discrepancy. Obviously, the Hölder regularity of financial time series is not constant in time, and this has important consequences : indeed, one can imagine that periods where the market is "quiet" correspond to high Hölder exponents, while low values of the exponent indicate sudden, "krach-like" variations. One then needs a process like $\mathrm{mBm}$ to model at the same time the long range dependence and a fluctuating regularity, and it is of obvious interest to have an expression for its autocovariance.

Traffic modeling :

Many Internet traces have been found to exhibit long range dependence. This has several consequences, for instance on the behaviors of the queues. Recently, it has been recognized that Internet traces also display some multifractal features, which are not compatible with a modeling by fBm. Among the other models that have been proposed, $\mathrm{mBm}$ has the advantage that it is a simple 
generalization of $\mathrm{fBm}$, and that, thanks to the analysis above, it allows to explain both the long range dependence and the wildly varying local regularity. With a right choice of $H(t)$, we can model accurately the multifractal properties of traffic traces, have the correct long range dependence exponent, and even accommodate the fact that different experiments sometimes give slightly different such exponents. This can be explained by non stationarity : depending on both the initial time and the unit lag, one will indeed observe different asymptotic behaviors. With the simple case investigated in Proposition 10, one can model a traffic where the long range dependence exponent exists in a generalized sense at all times and is an increasing function of the instant chosen for the beginning of the observations.

Finally, Proposition 4 could also serve as a basis for new estimation procedures, and for optimal sampling of $\mathrm{mBm}$.

\section{References}

[1] G.S. Ammar and W.B. Gragg, Superfast solution of real positive definitive Toeplitz systems, SIAM J. Matrix Anal. Appl., 9(1988), 61-76.

[2] A. Ayache, J. Lévy Véhel, The generalized Multifractional Brownian Motion, Théorème Limites et Longue Mémoire en Statistique, CIRM, Marseille, Novembre 1998.

[3] A. Benassi, S. Cohen, J. Istas, Identifying the multifractional function of a Gaussian process, Stat. and Prob. Letter 39, 337-345.

[4] A. Benassi, S. Jaffard, D. Roux, Gaussian processes and pseudodifferential elliptic operators, Rev. Mat. Iberoamericana, 13 (1), 19-89.

[5] J. Beran, Statistics for Long-Memory Processes (Chapman \& Hall, 1994).

[6] S. Cohen, From self-similarity to local self-similarity : the estimation problem, in Fractals: Theory and Applications in Engineering M. Dekking, J. Lévy Véhel, E. Lutton and C. Tricot (Eds). Springer Verlag, 1999.

[7] M.M. Daniel and A.S. Willski, Modeling and estimation of fractional Brownian motion using multiresolution stochastic processes, Fractals in Engineering, J. Lévy Véhel, E. Lutton and C. Tricot Eds (Springer Verlag 1997).

[8] P. Flandrin, On the spectrum of Fractional Brownian Motions, IEEE Tr. Inf. Theory, 35(1989), 197-199.

[9] M.S. Kesner, 1/f noises, Proc. IEEE, 70(1974), 212-218. 
[10] L.M. Kaplan, C.C.J. Kuo, An improved method for 2-D self-similar image synthesis, IEEE Tr. Image Proc., 5(1996), 754-761.

[11] R. Peltier, J. Lévy Véhel, Multifractional Brownian Motion: definition and preliminary results, Inria research report No. 2645, 1995.

[12] G.W. Wornell, Signal Processing with Fractals (Prentice Hall, 1996). 